\title{
Climate change: an enduring challenge for vector-borne disease prevention and control
}

\author{
Climate change is already affecting vector-borne disease transmission and spread, and its impacts are likely to \\ worsen. In the face of ongoing climate change, we must intensify efforts to prevent and control vector-borne diseases.
}

\section{Joacim Rocklöv and Robert Dubrow}

he rapid warming of the Earth, caused by anthropogenic greenhouse gas emissions, has profound long-term implications for the prevention and control of vector-borne diseases. Put simply, vectors, which are ectotherms (that is, cold-blooded animals), do better in a warmer world. Here, we discuss how climate affects the transmission dynamics and geographic spread of vector-borne diseases and the impact our changing climate has had thus far. We emphasize how the presence of many non-climate drivers of vector-borne disease transmission makes it difficult to isolate the role of climate change. We highlight the symbiotic intersection of (1) observational studies that elucidate how meteorological variables affect the incidence, transmission-season duration and spread of vector-borne diseases and (2) scenario-based modeling of the effects of future climate change that can aid long-term planning for the prevention and control of vector-borne diseases. Finally, to address the adverse impacts of climate change, we call for urgent and rapid reductions in greenhouse gas emissions, as well as adaptation to ongoing climate change through intensification of vector-borne disease prevention and control efforts.

\section{Climate change}

Anthropogenic greenhouse gas emissions have caused the mean global temperature to increase by $1{ }^{\circ} \mathrm{C}$ above preindustrial levels ${ }^{1,2}$. The impacts of a $1{ }^{\circ} \mathrm{C}$ rise have been profound, including a decrease in the number of cold days and nights, an increase in the number of warm days and nights, an increase in extreme heat events, decreased snow cover, and accelerating sea level rise. Global warming has exhibited considerable heterogeneity, with greater warming over land than over the oceans, the greatest warming occurring in the Arctic, and evidence for greater warming in winter versus summer and in nighttime versus daytime. Increased evaporation from warming has resulted in complex, region-specific changes in the hydrological cycle; while there has been an increase in overall global precipitation, some areas have become wetter and others have become drier. Both wet and dry regions, however, have experienced an increase in extreme precipitation events.

If current greenhouse gas emission trends continue, the mean global temperature could increase by 4 to $5^{\circ} \mathrm{C}$ above preindustrial levels by the end of the century $y^{2,3}$, which would result in dramatic intensification of the changes already observed. According to the authoritative Intergovernmental Panel on Climate Change (IPCC), "Continued emission of greenhouse gases will cause further warming and long-lasting changes in all components of the climate system, increasing the likelihood of severe, pervasive and irreversible impacts for people and ecosystems"3. The impact of climate change on the incidence, transmission season duration and spread of vector-borne diseases represents a major threat ${ }^{4}$.

\section{Vector-borne diseases}

A vector is an organism (most often an arthropod) that transmits an infectious pathogen from an infected human or animal host to an uninfected human. The World Health Organization identifies the major global vector-borne diseases as malaria, dengue, chikungunya, yellow fever, Zika virus disease, lymphatic filariasis, schistosomiasis, onchocerciasis, Chagas disease, leishmaniasis and Japanese encephalitis (Table 1$)^{5}$. Other vector-borne diseases of regional importance include African trypanosomiasis, Lyme disease, tick-borne encephalitis and West Nile fever. Tropical and subtropical low- and middle-income countries bear the highest burden of vector-borne diseases. Eight vector-borne diseases are considered to be neglected tropical diseases (Table 1).

Humans serve as the primary host for some vector-borne diseases, including malaria, dengue, chikungunya and Zika virus disease, whereas other vector-borne diseases have more complex transmission dynamics, with both human and non-human hosts (Table 1). For example, for Lyme disease, small mammals and birds serve as competent (reservoir) hosts (they are infected by the tick vector and they can infect ticks); deer and other large- and medium-sized mammals serve as incompetent hosts (they provide adult ticks with blood meals but do not become infected); and humans serve as dead-end hosts (they are infected by ticks but do not infect ticks). For West Nile virus, a wide range of bird species serve as reservoir hosts with a range of competence, whereas humans, horses and other mammals serve as dead-end hosts.

Among vector-borne diseases, malaria is the major killer, causing an estimated 620,000 deaths in 2017 (most occurring in Africa), followed by dengue, with an estimated 40,500 deaths (most occurring in Asia $)^{6}$. The estimated number of incident cases in 2017 was 209 million for malaria and 105 million for dengue ${ }^{7}$. While most other vector-borne neglected tropical diseases are seldom fatal, they involve chronic infections that confer substantial disability. In 2017, an estimated 65 million people were living with lymphatic filariasis, 143 million with schistosomiasis, 21 million with onchocerciasis, 6.2 million with Chagas disease and 4.1 million with leishmaniasis ${ }^{7}$.

\section{How climate affects vector-borne diseases}

Climate can affect the transmission dynamics, geographic spread and re-emergence of vector-borne diseases through multiple pathways, including direct effects on the pathogen, the vector, non-human hosts and humans. In addition to having direct effects on individual species, climate change can alter entire ecosystem habitats (including urban habitats), in which vectors or non-human hosts may thrive or fail.

Because arthropods and other vectors are ectotherms, it is expected that vector abundance, survival and feeding activity will increase with increasing temperature, as will the rate of development of the pathogen 


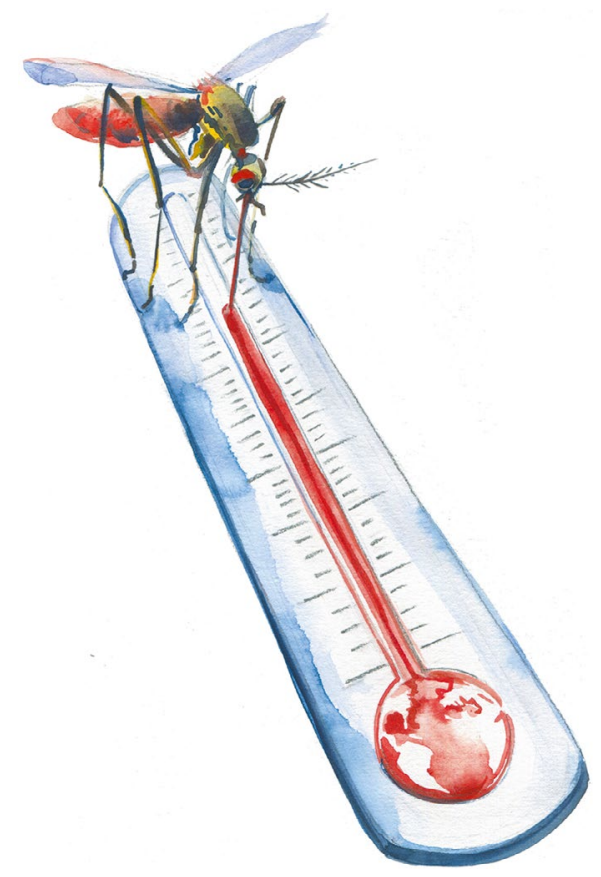

Credit: Chris Sharp

within the vector. Thus, the extrinsic incubation period (the time between ingestion of the pathogen by the vector and the vector becoming infective) for the dengue virus has been found to be inversely associated with ambient temperature ${ }^{8}$. In fact, an infected vector may never become competent (capable of transmitting infection) if the rate of development of the pathogen is so slow or survival of the vector is so short that the vector dies before becoming infective.

Although 'warmer is better' for vectors in general, relationships between temperature and vector survival, abundance and feeding behavior are often complex. For example, in the laboratory, survival of the dengue vector Aedes aegypti from egg to adult increases roughly linearly from near $0 \%$ at $15{ }^{\circ} \mathrm{C}$ to about $90 \%$ at $20^{\circ} \mathrm{C}$ and then slowly falls to about $60 \%$ at $35^{\circ} \mathrm{C}$; the time of development from egg to adult drops sharply from about 60 days at $15^{\circ} \mathrm{C}$ to 12 days at $20^{\circ} \mathrm{C}$ and then declines further to about 6 days at $27-34^{\circ} \mathrm{C}$; and the percentage of mosquitoes that complete a blood meal within 30 minutes after a host is made available plateaus at about $50 \%$ between $22^{\circ} \mathrm{C}$ and $28^{\circ} \mathrm{C}$ and then declines to almost $0 \%$ at $33^{\circ} \mathrm{C}$ ( ref. $^{9}$ ).

Clearly, the geographic range of $A$. aegypti (and other vectors) is limited by cooler ambient temperatures. As the Earth warms, the concerns are that the mosquito and virus will spread to higher latitudes and altitudes, that incidence will increase, and that the transmission season will lengthen in some endemic areas. (Aedes albopictus, the other dengue vector, has already exhibited widespread expansion into more temperate regions; however, the role of climate change is unclear.) There is also the possibility of a decrease in the incidence of dengue or other vector-borne diseases in endemic areas if they become so hot that vector survival or feeding are inhibited. Such areas, however, would still face other severe impacts from extreme heat.

The relationship between precipitation and vector abundance is complex and context specific ${ }^{9}$. Increased precipitation could provide more vector breeding sites; however, drought could also provide more breeding sites due to an increase in the use of containers for rainwater collection and storage - prime breeding sites for $A$. aegypti. Vector abundance could also be influenced by ecosystem change (for which climate change is a driver), which could degrade or enhance vector habitats and species competition, or it could increase or reduce the abundance of vector predators or vector pathogens.

Abundance and behaviors of both non-human and human hosts may be influenced by climate. Climate can influence a non-human host directly, or it can do so indirectly through ecosystem change, which can affect the abundance of food sources, predators and pathogens, making habitats either more or less hospitable. For example, birds serve as the reservoir host for West Nile virus. The shift in bird migration patterns and decline in bird populations in North America, caused by several factors - including climate change ${ }^{10}$ - may affect transmission of the virus from Culex mosquitoes to humans. Human population displacement caused by climate change can spread the vector or pathogen to new locations or can put immunologically susceptible populations in contact with the vector and pathogen.

\section{Climate change and vector-borne diseases}

There is no doubt that a suitable climate is necessary for the persistence or emergence of a vector-borne disease. Thus, although travel-acquired cases of dengue occur in Sweden, dengue is not endemic to Sweden because neither the Aedes mosquito nor the virus are established there, due to unsuitable climatic conditions. However, there also are many non-climate drivers that determine whether a vector-borne disease is found in a particular geographic area (Table 2). For example, malaria was present in the southern United States until the 1940s, when it was eradicated by aggressive vector control measures. Thus, a suitable climate is necessary but often not sufficient, as the climate determines the potential geographic distribution of a vector-borne disease, but other factors determine the actual distribution within the boundaries set by climate.

Non-climate drivers can be categorized into four groups: globalization and environment, sociodemographics, public health systems, and vector and pathogen characteristics $^{11}$ (Table 2). Despite climate change, during the past decade, substantial progress has been made against many vector-borne diseases. This progress has been attributed to sociodemographic (for example, economic development) and public health system (for example, vector control and other public health interventions) drivers ${ }^{5}$. Between 2007 and 2017, the age-standardized disability-adjusted life year (DALY) rates decreased by $39 \%$ for malaria, $21 \%$ for yellow fever, $45 \%$ for lymphatic filariasis, $29 \%$ for schistosomiasis, $8 \%$ for onchocerciasis, $23 \%$ for Chagas disease, $56 \%$ for leishmaniasis and $83 \%$ for African trypanosomiasis ${ }^{12}$.

Thus, economic development and public health interventions - and not climate change - appear to have been the primary drivers of the incidence of these vector-borne diseases globally over the past decade. It is possible that progress would have been even greater in the absence of climate change, but this would be difficult to convincingly demonstrate. Vector-borne disease drivers, including climate change, can affect and interact with each other, and the effect of one driver may be mediated through processes linked to other drivers, making it difficult to isolate driver effects and thus to unambiguously attribute changes in transmission or spread to climate change. An additional problem in disentangling climate change from other effects is a lack of high-quality global-scale observational data on disease incidence and non-climate drivers.

Dengue is the only major global vector-borne disease for which the age-standardized DALY rate increased between 2007 and 2017, and it increased by $26 \%{ }^{12}$. This trend represents a continuation of the explosive expansion of dengue since the 1950s, driven by urbanization, globalization and ineffective mosquito control $^{13}$. Although climate has set geographic limits on dengue expansion, it would be difficult to demonstrate that climate change has been a contributing factor on a global scale.

In summary, climate represents a critical factor in determining vector-borne disease incidence, but it does not appear that climate 
Table 1 | Major global and regional vector-borne diseases

\begin{tabular}{|c|c|c|c|}
\hline Disease & Pathogen & Primary vector(s) & $\begin{array}{l}\text { Primary non-human reservoir } \\
\text { (competent) hosts }\end{array}$ \\
\hline Malaria & Plasmodium parasite & Anopheles mosquito & Non-human hosts of minor concern \\
\hline Dengue $^{\star}$ & Flavivirus & $\begin{array}{l}\text { Aedes aegypti and Aedes albopictus } \\
\text { mosquitoes }\end{array}$ & Non-human hosts of minor concern \\
\hline Yellow fever & Flavivirus & A. aegypti and A. albopictus mosquitoes & Non-human primates \\
\hline Zika & Flavivirus & A. aegypti and A. albopictus mosquitoes & Non-human hosts of minor concern \\
\hline Chikungunya* & Alphavirus & A. aegypti and A. albopictus mosquitoes & Non-human hosts of minor concern \\
\hline Lymphatic filariasis ${ }^{\star}$ & Various filarial nematodes & A variety of mosquito genera & Non-human hosts of minor concern \\
\hline Schistosomiasis* & Schistosoma trematode & Snail & Non-human hosts of minor concern \\
\hline Onchocerciasis ${ }^{\star}$ & Onchocerca volvulus nematode & Simulium (black fly) & None \\
\hline Chagas disease $^{\star}$ & Trypanosoma cruzi parasite & Triatomine bug & Mammals \\
\hline Leishmaniasis* & Leishmania parasite & Sand fly & Rodents, dogs, other mammals \\
\hline Japanese encephalitis & Flavivirus & Culex mosquitoes & Pigs, birds \\
\hline African trypanosomiasis* & Trypanosoma brucei parasite & Glossina (tsetse fly) & Wild and domestic animals \\
\hline Lyme disease & Borrelia spirochete & Ixodes ticks & $\begin{array}{l}\text { White-footed mouse and other small } \\
\text { mammals, birds }\end{array}$ \\
\hline Tick-borne encephalitis & Flavivirus & Ixodes ticks & Small rodents \\
\hline West Nile fever & Flavivirus & Culex mosquitoes & Birds \\
\hline
\end{tabular}

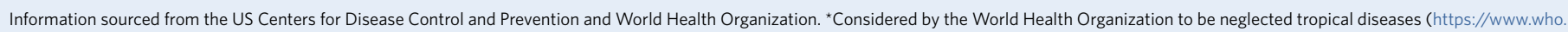
int/neglected_diseases/diseases/summary/en/).

change has been a primary driver thus far on a global scale. Nevertheless, there are serious reasons for concern. First, there have been numerous regional or local signals that climate change is affecting or will be likely to affect vector-borne disease incidence or spread. Second, one would expect a time lag between changes to the climate that make a non-endemic area suitable for endemicity, actual establishment of the vector and pathogen in that area, and occurrence and reporting of cases of disease. Third, climate change could emerge as a synergistic driver if there were backsliding on vector control measures and other public health efforts due to complacency or a lack of political will. Fourth, one would expect pathogen and vector evolution to accelerate in a warming world due to higher replication rates. Such evolution can play a significant role in vector-borne disease emergence, re-emergence and spread (for example, through pathogens evolving resistance to treatment or vectors evolving resistance to pesticides). Finally, climate change will only get worse in the foreseeable future, with models projecting a substantial expansion of regions with a suitable climate for a number of vector-borne diseases.

\section{Regional and local signals of climate change effects on vector-borne diseases} There are many regional and local signals that climate change has already affected or is likely to affect vector-borne disease transmission or spread. For example, a time-series analysis of monthly malaria cases in the highlands of Colombia and Ethiopia provided evidence for a shift in the altitudinal distribution of malaria toward higher altitudes in warmer years, suggesting that, in the absence of intervention, the malaria burden will increase at higher elevations as the climate warms ${ }^{14}$.

Pathogens may be dispersed into non-endemic localities through travel, trade or migration. The number of dengue cases imported into Europe is highly correlated with the number of travelers arriving from endemic zones ${ }^{15}$. Local (autochthonous) outbreaks of dengue and chikungunya have occurred in southern Europe when infected travelers pass the pathogen to the mosquito vector A. albopictus (now established in southern Europe), which in turn causes secondary cases of human infection ${ }^{16}$. Although such outbreaks are still rare, and the current risk of sustained outbreaks is low, as the climate warms, vigilance is needed to prevent sustained outbreaks or establishment of endemicity in the future.

Vectorial capacity (a summary measure of the capacity of a vector to transmit disease that integrates information on vector abundance, survival, competence and feeding rate, and the length of the extrinsic incubation period), which is dependent on temperature conditions, helped explain the
2017 chikungunya outbreaks transmitted by A. albopictus in Europe ${ }^{17}$ and the 2015-2016 Zika virus epidemic transmitted by $A$. aegypti and A. albopictus in South America ${ }^{18}$. In the latter case, temperature conditions associated with the 2015-2016 El Niño/ Southern Oscillation were exceptionally conducive to Zika virus transmission. Globally, vectorial capacity for both dengue vectors (A. aegypti and A. albopictus) has been rising steadily since the 1980s, with nine of the ten highest years occurring since $2000^{19}$.

In the United States, higher cumulative growing degree days (a temperature metric), lower cumulative precipitation and lower saturation deficit (inversely related to humidity) were found to be associated with an earlier start to the Lyme disease season ${ }^{20}$. Higher temperature was found to be the most important determinant of environmental suitability for the establishment of the Lyme disease Ixodes tick vector in southern Canada, where it has been spreading ${ }^{21}$. Milder and shorter winters in Quebec, Canada are associated with the northern spread of the white-footed mouse, the primary reservoir host for the Lyme disease pathogen Borrelia burgdorferi ${ }^{22}$.

\section{Future climate change and vector-borne diseases}

Regional and local signals of the effect of climate change on vector-borne diseases 


\section{Table 2 | Non-climate drivers of the transmission and spread of vector-borne diseases}

\section{Globalization and environment}

Driver

Deforestation, mining and dams

Ecosystem degradation/change

International travel and trade

Urbanization

Population displacement

\section{Sociodemographic factors}

Driver

Population demographic composition

Level of economic development

Baseline incidence of disease

Population health status

Humanitarian crises

Public health systems

Driver

Surveillance

Early warning systems

Vector control

Quality of healthcare system

Research

\section{Vector and pathogen characteristics}

Driver

Insecticide resistance

Vector evolution

Pathogen drug resistance

Pathogen evolution

indicate the need for vigilance. To address the vector-borne disease threat, along with the many other severe risks posed by climate change, the world must meet the Paris Climate Agreement goal of limiting global warming to no more than $1.5^{\circ} \mathrm{C}$ above preindustrial levels. This will require immediate and steep reductions in greenhouse gas emissions through rapid and far-reaching changes in energy, land use, transportation, urban, built environment, food and industrial systems ${ }^{1}$.

However, our planet has already warmed by $1{ }^{\circ} \mathrm{C}$, and limiting warming to another $0.5^{\circ} \mathrm{C}$ is the absolute best we can do. In fact, greenhouse gas emissions continue to rise, and there is no evidence of worldwide political commitment to appreciably veer from the current 'business-as-usual' path that could lead to a $4-5^{\circ} \mathrm{C}$ rise by 2100 . Prudence requires that we plan for a range of possible futures.

First, we need to continue to conduct observational studies, similar to the ones described in the previous section, to understand the relationships between meteorological variables and vector-borne disease incidence and spread. These studies usually involve applying mathematical or statistical models, which can be process-based (taking into account fundamental biological processes and mechanisms involved in transmission dynamics) or empirical (based on observed statistical associations, without attempting to incorporate mechanisms). Valid models depend on high-quality, long-term observational data. Models are disease-specific and are often valid for only a specific geographic location or time period.

Due to difficulties in accurately estimating mechanistic parameters, empirical models tend to be more feasible. Typically, these are time series models that incorporate latency times between meteorological exposures and the disease outcome. For example, a study in Sri Lanka found dengue risk to be highest six to ten weeks following precipitation of more than $300 \mathrm{~mm}$ per week ${ }^{23}$. A study in Singapore found that weekly mean temperature lagged by up to 16 weeks, weekly cumulative precipitation lagged by up to 16 weeks, and season, trend of dengue cases and past dengue cases explained $84 \%$ of the variance in dengue distribution ${ }^{24}$. Thus, past disease incidence and other non-climate predictors (for example, population density) should be evaluated for potential inclusion in models.

Predicting how future climate change will affect vector-borne diseases is not possible because there are too many uncertainties. First, how the climate will change will depend on the aggressiveness of human actions to reduce greenhouse gas emissions. Second, prediction would need to take into account changes in non-climate drivers, many of which are also unpredictable. For example, how can we predict the pace of development of more effective public health interventions (for example, vector control and vaccine development) or the political will needed for sustained efforts to control vector-borne diseases? Trends in these and other drivers are likely to be non-linear.

Although prediction is not possible, considerable effort has been devoted to developing models based on alternative scenarios to gain an understanding of a range of possible futures. Projections about the future incidence and distribution of a specific vector-borne disease are made by linking a future climate change scenario (based on projected greenhouse gas emissions) with a process-based or empirical vector-borne disease model that has been validated using historical observational data. The simplest approach is to do this without incorporating scenarios for non-climate drivers such as travel, socioeconomic factors or public health advances. This approach asks: if the climate were to change according to a particular scenario, but non-climate drivers were to remain the same, what would 
be the effect on the vector-borne disease in question? Although simple, this approach can help inform long-term planning, according to the precautionary principle.

The IPCC has developed four Representative Concentration Pathway (RCP) scenarios for greenhouse gas emissions, ranging from a high-emission 'business-as-usual' scenario (RCP8.5) to a low-emission, aggressive mitigation scenario (RCP2.6). For a given RCP, climate models, termed general circulation models (GCMs), are used to project temperature, precipitation and humidity to 2100 and beyond. Typically, an ensemble of different GCMs is used to assess model uncertainty. In turn, these meteorological projections can be used in a vector-borne disease model to project changes in incidence or distribution. For example, under RCP8.5, projected changes in temperature and diurnal temperature range in temperate Northern Hemisphere regions are projected to result in large increases in the vectorial capacity of A. aegypti for dengue transmission by $2100^{8}$.

Models can also incorporate non-climate drivers. In addition to RCPs, the IPCC has developed five Shared Socioeconomic Pathways (SSPs), which describe alternative scenarios of socioeconomic change up through 2100. Studies have made vector-borne disease projections based on combined RCP-SSP scenarios. In one global study, future dengue occurrence up through 2080 was found to be highly dependent on the RCP-SSP scenario used ${ }^{25}$.

A study on the impact of climate change on global malaria distribution forecasted changes in the length of the malaria transmission season and in the populations at risk of contracting malaria for each of the four RCPs, using an ensemble of five GCMs and five malaria models (some process-based and some empirical) ${ }^{26}$. SSP2 population projections were incorporated into the model. Comparing 2069-2099 with 1980-2010, the modeling found consistent increases in the length of the malaria transmission season in highland regions around the world, along with consistent decreases in tropical regions. The net effect of future climate change on future populations at risk was relatively small, although there were large regional differences.

Another study used empirical global mosquito distribution data, meteorological variables and measures of urbanization and human mobility to build a model of historical spatial spread for $A$. aegypti and $A$. albopictus, and used it to project future spread until 2080 under different
RCP scenarios. The investigators found that historical spread of these vectors, as well as spread over the next 5-15 years, could be explained by human movement patterns, whereas in later years, expansion will be driven by climate change (especially temperature increases) and urbanization, with higher emission scenarios associated with greater expansion ${ }^{27}$.

Thus, empirical models that incorporate multiple predictors can help identify key historical drivers, and scenario-based models that incorporate multiple drivers can provide an indication of future key drivers. We can look forward to several future improvements to this modeling approach. First, valid empirical modeling, on which scenario modeling is based, is crucially dependent on high-quality, long-term datasets on vector-borne disease incidence, vector and animal host populations, non-climate drivers and meteorological variables. We need to develop more such local and regional datasets. Second, we need to improve methods for quantifying difficult-to-measure non-climate predictors, such as land and water use, ecosystem change and population displacement, and then incorporate these predictors into empirical models to identify additional key historical drivers, which could then be included in scenario-based models. Third, we need to test the effects of plausible scenarios of non-linear, abrupt changes in climate or non-climate drivers.

Finally, we need to intensify prevention and control efforts, including controlling vectors, diagnosing and treating diseases early (to remove sources of infection available to feeding vectors), vaccinating, improving water and sanitation systems, cooling urban heat islands, and other interventions. Efforts are underway to develop short-term (that is, weeks to months) vector-borne disease models of sufficient validity to be used in early warning systems, which would prompt timely public health measures to prevent impending outbreaks ${ }^{28}$. Incorporation of a range of intervention scenarios into scenario modeling could help inform optimization of both current and future combinations of interventions that will meet the enduring challenges of prevention and control of vector-borne diseases in the face of ongoing climate change.

\section{Joacim Rocklöv $v^{1,4}$ and Robert Dubrow (D) 2,3,4凶}

${ }^{1}$ Department of Public Health and Clinical Medicine, Umeå University, Umeå, Sweden. ${ }^{2}$ Department of Environmental Health Sciences, Yale School of Public
Health and Yale School of Medicine, Yale University, New Haven, CT, USA. ${ }^{3}$ Yale Center on Climate Change and Health, Yale University, New Haven, CT, USA. ${ }^{4}$ These authors contributed equally: Joacim Rocklöv, Robert Dubrow.

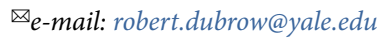

Published online: 20 April 2020

https://doi.org/10.1038/s41590-020-0648-y

References

1. IPCC. Special Report on Global Warming of $1.5^{\circ} \mathrm{C}$ (eds Masson-Delmotte, V. et al.) (WMO, 2018).

2. Wuebbles, D. J. et al. (eds). Climate Science Special Report: Fourth National Climate Assessment, Volume I (US Global Change Research Program, 2017).

3. IPCC. Climate Change 2014: Synthesis Report (eds Core Writing Team, Pachauri, R. K. \& Meyer, L. A.) (IPCC, 2014).

4. IPCC. Climate Change 2014: Impacts, Adaptation, and Vulnerability. Part A: Global and Sectoral Aspects (eds Fields, C. B. et al.) (Cambridge Univ. Press, 2014)

5. Global Vector Control Response 2017-2030 (World Health Organization, 2017).

6. GBD 2017 Causes of Death Collaborators. Lancet 392, 1736-1788 (2018).

7. GBD 2017 Disease and Injury Incidence and Prevalence Collaborators. Lancet 392, 1789-1858 (2018).

8. Liu-Helmersson, J., Stenlund, H., Wilder-Smith, A. \& Rocklöv, J. PLoS ONE 9, e89783 (2014).

9. Morin, C. W., Comrie, A. C. \& Ernst, K. Environ. Health Perspect. 121, 1264-1272 (2013).

10. Rosenberg, K. V. et al. Science 366, 120-124 (2019).

11. Semenza, J. C. et al. Emerg. Infect. Dis. 22, 581-589 (2016).

12. GBD 2017 DALYs and HALE Collaborators. Lancet 392, 1859-1922 (2018).

13. Gubler, D. J. Trop. Med. Health 39(4 Suppl.), 3-11 (2011).

14. Siraj, A. S. et al. Science 343, 1154-1158 (2014).

15. Semenza, J. C. et al. PLoS Negl. Trop. Dis. 8, e3278 (2014)

16. Lillepold, K., Rocklöv, J., Liu-Helmersson, J., Sewe, M. \& Semenza, J. C. J. Travel Med. 26, taz017 (2019).

17. Rocklöv, J. et al. Emerg. Infect. Dis. 25, 1041-1049 (2019).

18. Caminade, C. et al. Proc. Natl Acad. Sci. USA 114, 119-124 (2017).

9. Watts, N. et al. Lancet 394, 1836-1878 (2019).

20. Moore, S. M., Eisen, R. J., Monaghan, A. \& Mead, P. Am. J. Trop. Med. Hyg. 90, 486-496 (2014).

21. Leighton, P. A., Koffi, J. K., Pelcat, Y., Lindsay, L. R. \& Ogden, N. H. J. Appl. Ecol. 49, 457-464 (2012).

22. Roy-Dufresne, E., Logan, T., Simon, J. A., Chmura, G. L. \& Millien, V. PLoS ONE 8, e80724 (2013).

23. Liyanage, P. et al. Int. J. Environ. Res. Public Health 13, 1087 (2016).

24. Hii, Y. L., Zhu, H., Ng, N., Ng, L. C. \& Rocklöv, J. PLoS Negl. Trop. Dis. 6, e1908 (2012).

25. Messina, J. P. et al. Nat. Microbiol. 4, 1508-1515 (2019)

26. Caminade, C. et al. Proc. Natl Acad. Sci. USA 111, 3286-3291 (2014).

27. Kraemer, M. U. G. et al. Nat. Microbiol. 4, 854-863 (2019).

28. Johansson, M. A. et al. Proc. Natl Acad. Sci. USA 116, 24268-24274 (2019).

\section{Acknowledgements}

J.R. received funding from the Swedish Research Council Formas (grant nos. 2018-01754 and 2017-01300) and the ZikaPlan project funded by the European Union research and innovation program Horizon 2020 (grant agreement no. 734584). R.D. received funding from the High Tide Foundation.

\section{Author contributions}

The authors worked collaboratively and contributed equally to the article.

\section{Competing interests}

The authors declare no competing interests. 